\title{
Article
}

\section{Bounds for the Energy of Graphs}

\author{
Slobodan Filipovski ${ }^{1, *}$ (D) and Robert Jajcay ${ }^{2}$ \\ 1 FAMNIT, University of Primorska, 6000 Koper, Slovenia \\ 2 Department of Algebra and Geometry, Faculty of Mathematics, Physics and Informatics, \\ Comenius University, 84248 Bratislava, Slovakia; robert.jajcay@fmph.uniba.sk \\ * Correspondence: slobodan.filipovski@famnit.upr.si
}

check for updates

Citation: Filipovski, S.; Jajcay, R. Bounds for the Energy of Graphs. Mathematics 2021, 9, 1687. https:/ / doi.org/10.3390/math9141687

Received: 16 June 2021

Accepted: 13 July 2021

Published: 18 July 2021

Publisher's Note: MDPI stays neutral with regard to jurisdictional claims in published maps and institutional affiliations.

Copyright: (c) 2021 by the authors. Licensee MDPI, Basel, Switzerland. This article is an open access article distributed under the terms and conditions of the Creative Commons Attribution (CC BY) license (https:/ / creativecommons.org/licenses/by/ $4.0 /)$.

\begin{abstract}
Let $G$ be a graph on $n$ vertices and $m$ edges, with maximum degree $\Delta(G)$ and minimum degree $\delta(G)$. Let $A$ be the adjacency matrix of $G$, and let $\lambda_{1} \geq \lambda_{2} \geq \ldots \geq \lambda_{n}$ be the eigenvalues of $G$. The energy of $G$, denoted by $E(G)$, is defined as the sum of the absolute values of the eigenvalues of $G$, that is $E(G)=\left|\lambda_{1}\right|+\ldots+\left|\lambda_{n}\right|$. The energy of $G$ is known to be at least twice the minimum degree of $G, E(G) \geq 2 \delta(G)$. Akbari and Hosseinzadeh conjectured that the energy of a graph $G$ whose adjacency matrix is nonsingular is in fact greater than or equal to the sum of the maximum and the minimum degrees of $G$, i.e., $E(G) \geq \Delta(G)+\delta(G)$. In this paper, we present a proof of this conjecture for hyperenergetic graphs, and we prove an inequality that appears to support the conjectured inequality. Additionally, we derive various lower and upper bounds for $E(G)$. The results rely on elementary inequalities and their application.
\end{abstract}

Keywords: energy of graphs; conjecture; new bounds

\section{Introduction}

Let $G=(V, E)$ be a simple undirected graph with $n$ vertices $v_{1}, v_{2}, \ldots, v_{n}$ and $m$ edges. An adjacency matrix $A=A(G)$ of the graph $G$ is the square matrix $\left[a_{i j}\right]$ where $a_{i j}=1$ if the vertex $v_{i}$ is adjacent to the vertex $v_{j}$, and $a_{i j}=0$ otherwise. The eigenvalues $\lambda_{1}, \lambda_{2}, \ldots, \lambda_{n}$ of the matrix $A$ are called the eigenvalues of the graph $G$. Since $G$ is an undirected graph, the matrix $A$ is symmetric; consequently, its eigenvalues are real numbers. Moreover, $\operatorname{trace}(A)=0$, that is:

$$
\sum_{i=1}^{n} \lambda_{i}=\lambda_{1}+\lambda_{2}+\ldots+\lambda_{n}=0
$$

and:

$$
\operatorname{trace}\left(A^{2}\right)=\sum_{i=1}^{n} \lambda_{i}^{2}=2 m .
$$

I. Gutman in [1] defined the energy of $G, E(G)$, as the sum of the absolute values of the eigenvalues of $A$. Mathematically,

$$
E(G)=\sum_{i=1}^{n}\left|\lambda_{i}\right|
$$

It is known that the energy of a given graph can be used to approximate the $\pi$-electron energy of a molecule. Thus, the graph energy is closely related to the concepts of theoretical chemistry; see [2-6]. The parameter $E(G)$ and its bounds were intensively studied in the decades before. For example, the reader is referred to [6-11].

In 1971, McClelland [6] obtained the first graph-energy result addressing the upper bound for $E(G)$ involving the order $n$ and the size $m$ :

$$
E(G) \leq \sqrt{2 m n}
$$


The bound in (4) was improved by Koolen and Moulton [10]. They proved, if $G$ is a graph of order $n$ and size $m$ and if $2 m>n$, then:

$$
E(G) \leq \frac{2 m}{n}+\sqrt{(n-1)\left(2 m-\left(\frac{2 m}{n}\right)^{2}\right)} .
$$

In [6], McClelland also derived a lower bound for $E(G)$ in terms of $n, m$ and $\operatorname{det}(A)$ (the determinant of the adjacency matrix $A$ ):

$$
E(G) \geq \sqrt{2 m+n(n-1)|\operatorname{det}(A)|^{\frac{2}{n}}}
$$

Almost thirty years later, a simpler lower bound involving only the number of edges $m$ was obtained in [12]:

$$
E(G) \geq 2 \sqrt{m}
$$

In 2019, Ma proved in [13] that the energy of a graph $G$ is greater than or equal to twice the minimum degree of $G$, that is,

$$
E(G) \geq 2 \delta(G)
$$

This result was recently independently re-proven in a shorter way in [14,15]. Moreover, in [15], Akbari and Hosseinzadeh stated the following conjecture, which presents an improvement on the bound (8):

Conjecture 1. For every graph with maximum degree $\Delta(G)$ and minimum degree $\delta(G)$ whose adjacency matrix is nonsingular, $E(G) \geq \Delta(G)+\delta(G)$, and the equality holds if and only if $G$ is a complete graph.

In a recent paper [16], Akbari et al. proved the validity of this conjecture for planar graphs, triangle-free graphs, and quadrangle-free graphs. In this paper, we consider the above conjecture and prove it for special families of graphs. Furthermore, we obtain new bounds for the energy of $G$, in terms of $n$ and $\operatorname{det}(A)$, when $G$ is a reciprocal graph and when the spectrum of $G$ contains exactly one positive eigenvalue. We show that some of our results are better than the well-known upper bounds.

\section{Lower Bounds for the Energy of Graphs}

One possible way to attack Conjecture $1, E \geq \Delta+\delta$, is to obtain an upper bound for $\Delta+\delta$ and then compare it with a lower bound for $E$. Since $\delta \leq \frac{2 m}{n}$, to obtain an upper bound on $\Delta+\delta$, in Proposition 1, we focus on obtaining an upper bound for $\Delta$ using again the parameters $n, m$ and the first Zagreb index $M_{1}$. Recall that if $G$ is a graph of order $n$ having degrees $d_{1}, \ldots, d_{n}$, the first Zagreb index of $G$, denoted by $M_{1}(G)$, is the sum of squares of its vertex degrees:

$$
M_{1}(G)=\sum_{i=1}^{n} d_{i}^{2}=d_{1}^{2}+d_{2}^{2}+\ldots+d_{n}^{2} .
$$

Many bounds exist for the first Zagreb index. In this paper, we use the following result, which appeared in [17]:

Theorem 1. Let $G$ be a simple connected graph with $n$ vertices and $m$ edges. Then:

$$
M_{1}(G) \geq \frac{4 m^{2}}{n} .
$$

Equality holds if and only if $G$ is regular. 
As promised above, our next proposition will provide us with an upper bound on the maximal degree. It is easy to see that this proposition is only interesting when $G$ is not regular.

Proposition 1. Let $G$ be a graph with $n$ vertices, $m$ edges, and the first Zagreb index $M_{1}$. The maximum degree of $G, \Delta(G)$, satisfies the inequality:

$$
\Delta(G) \leq \frac{2 m}{n}+\frac{n-1}{n} \sqrt{\frac{n M_{1}-4 m^{2}}{n-1}} .
$$

Proof. Applying the inequality between the quadratic and the arithmetic means to the degrees $d_{2}, \ldots, d_{n}$ provides us with the inequality:

$$
M_{1}=d_{1}^{2}+d_{2}^{2}+\ldots+d_{n}^{2} \geq \Delta^{2}+\frac{\left(d_{2}+\ldots+d_{n}\right)^{2}}{n-1}=\Delta^{2}+\frac{(2 m-\Delta)^{2}}{n-1} .
$$

From (11), we obtain the quadratic inequality in $\Delta$ :

$$
f(\Delta)=n \Delta^{2}-4 m \Delta+4 m^{2}-(n-1) M_{1} \leq 0 .
$$

By computing the discriminant of $f(\Delta)$, we obtain:

$$
D(f(\Delta))=16 m^{2}-4 n\left(4 m^{2}-(n-1) M_{1}\right)=4(n-1)\left(n M_{1}-4 m^{2}\right) \geq 0 .
$$

Therefore,

$$
f(\Delta) \leq 0 \Leftrightarrow \Delta \in\left[\Delta_{1}, \Delta_{2}\right] .
$$

Now, solving the equation $n \Delta^{2}-4 m \Delta+4 m^{2}-(n-1) M_{1}=0$, we obtain:

$$
\Delta_{1,2}=\frac{2 m}{n} \pm \frac{n-1}{n} \sqrt{\frac{n M_{1}-4 m^{2}}{n-1}} .
$$

The next theorem follows from Proposition 1.

Theorem 2. Let $G$ be a graph with $n$ vertices, $m$ edges, maximum degree $\Delta$, minimum degree $\delta$, and first Zagreb index $M_{1}$. Then,

$$
E(G) \geq \Delta+\delta-\frac{n-1}{n} \sqrt{\frac{n M_{1}-4 m^{2}}{n-1}}
$$

Proof. The relation $\delta \leq \frac{2 m}{n}$ and Proposition 1 yield:

$$
\Delta+\delta \leq \frac{2 m}{n}+\frac{n-1}{n} \sqrt{\frac{n M_{1}-4 m^{2}}{n-1}}+\frac{2 m}{n}=\frac{4 m}{n}+\frac{n-1}{n} \sqrt{\frac{n M_{1}-4 m^{2}}{n-1}} .
$$

The inequality (13) follows easily from $E(G) \geq \frac{4 m}{n}$.

Remark 1. Recall that $M_{1} \geq \frac{4 m^{2}}{n}$ (with equality occurring only for regular graphs) [17]. In the case when the difference between the Zagreb index of a graph $G$ and the lower bound $\frac{4 m^{2}}{n}$ is small, specifically, when the value $\frac{n-1}{n} \sqrt{\frac{n M_{1}-4 m^{2}}{n-1}}$ is small, Theorem 2 appears to support Conjecture 1 of Akbari and Hosseinzadeh. In particular, should the sequence of the first Zagreb indices of an 
infinite family of graphs $\left\{G_{n}\right\}_{n=1}^{\infty}$ tend to $\frac{4 m^{2}}{n}$, the lower bound for $E\left(G_{n}\right)$ shown in Theorem 2 tends to $\Delta+\delta$ :

$$
\Delta+\delta-\frac{n-1}{n} \sqrt{\frac{n M_{1}-4 m^{2}}{n-1}} \rightarrow \Delta+\delta .
$$

Let $\frac{\Delta}{\delta}=t \geq 1$. In the next result, we derive a lower bound for $E(G)$ in terms of $n$ and $t$. Since $E(G) \geq 2 \sqrt{m}$, it suffices to obtain a lower bound for the size of the graph in terms of $n$ and $t$. We use the following result published in [18].

Theorem 3. Let $G$ be a connected $(n, m)$-graph. Then:

$$
M_{1}(G) \leq \frac{2 m^{2}}{n}+\left(\frac{\Delta}{\delta}+\frac{\delta}{\Delta}\right) \frac{m^{2}}{n}
$$

with equality if and only if $G$ is a regular graph or $G$ is a bidegreed graph such that $\Delta+\delta$ divides $\delta n$ and there are exactly $p=\frac{2 n}{\Delta+\delta}$ vertices of degree $\Delta$ and $q=\frac{n \Delta}{\Delta+\delta}$ vertices of degree $\delta$.

From Proposition 1 and Theorem 3, we have:

Theorem 4. Let $G$ be a graph on $n$ vertices and $m$ edges with maximum degree $\Delta \geq 4$, and minimum degree $\delta \geq 1$. If $t=\frac{\Delta}{\delta}$, then:

$$
E(G) \geq 4 \sqrt{\frac{n}{2+\sqrt{(n-1)\left(t+\frac{1}{t}-2\right)}}} .
$$

Proof. Let $M_{1}$ be the first Zagreb index of G. From (15), we obtain:

$$
n M_{1}-4 m^{2} \leq m^{2}\left(t+\frac{1}{t}-2\right) \text {. }
$$

Thus:

$$
\frac{n-1}{n} \sqrt{\frac{n M_{1}-4 m^{2}}{n-1}} \leq \frac{m}{n} \sqrt{(n-1)\left(t+\frac{1}{t}-2\right)} .
$$

By using Proposition 1:

$$
\Delta \leq \frac{2 m}{n}+\frac{m}{n} \sqrt{(n-1)\left(t+\frac{1}{t}-2\right)} .
$$

The inequality (16) implies:

$$
m \geq \frac{n \Delta}{2+\sqrt{(n-1)\left(t+\frac{1}{t}-2\right)}} \geq \frac{4 n}{2+\sqrt{(n-1)\left(t+\frac{1}{t}-2\right)}}
$$

Now, from (17) and from the relation $E(G) \geq 2 \sqrt{m}$, we obtain the desired lower bound for $E(G)$.

Remark 2. From $2 m \geq n \delta$, we have $m \geq \frac{n \delta}{2} \geq \frac{n}{2}$. The reason why we take $\Delta \geq 4$ comes from the inequality $m \geq \frac{n \Delta}{2+\sqrt{(n-1)\left(t+\frac{1}{t}-2\right)}}$. Without any assumption for $\Delta$, we have $m \geq$ 
$\frac{n}{2+\sqrt{(n-1)\left(t+\frac{1}{t}-2\right)}}$, which is a weaker bound than the trivial bound $m \geq \frac{n}{2}$. Moreover, if the maximum degree $\Delta$ and the minimum degree $\delta$ are known, then:

$$
m \geq \frac{\Delta+(n-1) \delta}{2} \geq \frac{n \Delta}{2+\sqrt{(n-1)\left(\frac{\Delta}{\delta}+\frac{\delta}{\Delta}-2\right)}}
$$

In this case:

$$
E(G) \geq 2 \sqrt{m} \geq \sqrt{2(\Delta+(n-1) \delta)} \geq 2 \sqrt{\frac{n \Delta}{2+\sqrt{(n-1)\left(\frac{\Delta}{\delta}+\frac{\delta}{\Delta}-2\right)}}} .
$$

In the last two results of this section, we derive a lower bound for $E(G)$ in terms of $n$ and $\operatorname{det}(A)$ and a lower bound for $E(G)$ if $G$ is a reciprocal graph.

Proposition 2. Let $G$ be a graph on $n$ vertices with the largest eigenvalue $\lambda_{1}$. If $A$ is the adjacency matrix of $G$, then:

$$
E(G) \geq \lambda_{1}+\frac{((n-1) \sqrt[n]{|\operatorname{det}(A)|})^{2}}{(n-2) \sqrt[n]{|\operatorname{det}(A)|}+\lambda_{1}}
$$

Proof. At the Romanian Mathematical Olympiad in 1999 [19], it was proven that, if $x_{1}, \ldots$, $x_{n}$ are positive real numbers such that $x_{1} \cdots x_{n}=1$, then:

$$
\frac{1}{n-1+x_{1}}+\ldots+\frac{1}{n-1+x_{n}} \leq 1
$$

Let $\lambda_{1} \geq \ldots \geq \lambda_{n}$ be the eigenvalues of $G$. Assuming $x_{i}=\frac{\left|\lambda_{i}\right|}{\sqrt[n]{|\operatorname{det}(A)|}}$ for each $i=1, \ldots, n$, we have $x_{1} \cdots x_{n}=1$. From (19) and by using the inequality between arithmetic and harmonic means for the numbers $\frac{1}{n-1+\frac{\left|\lambda_{2}\right|}{\sqrt[n]{|\operatorname{det}(A)|}}}, \ldots, \frac{1}{n-1+\frac{\left|\lambda_{n}\right|}{\sqrt[n]{|\operatorname{det}(A)|}}}$, we obtain the inequality:

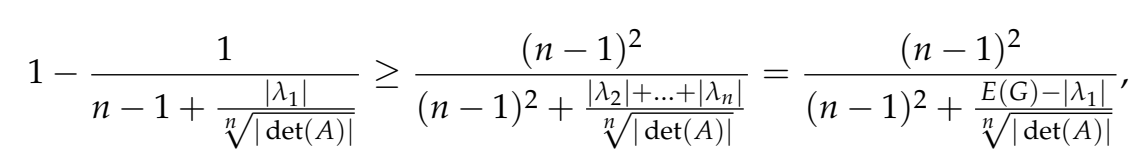

that is,

$$
\frac{(n-2) \sqrt[n]{|\operatorname{det}(A)|}+\left|\lambda_{1}\right|}{(n-1) \sqrt[n]{|\operatorname{det}(A)|}+\left|\lambda_{1}\right|} \geq \frac{(n-1)^{2} \sqrt[n]{|\operatorname{det}(A)|}}{(n-1)^{2} \sqrt[n]{|\operatorname{det}(A)|}+E(G)-\left|\lambda_{1}\right|}
$$

The last inequality is equivalent to:

$$
(n-1) 2 \sqrt[n]{|\operatorname{det}(A)|}+E(G)-\lambda_{1} \geq \frac{(n-1) \sqrt[n]{|\operatorname{det}(A)|}\left((n-1) \sqrt[n]{|\operatorname{det}(A)|}+\lambda_{1}\right)}{(n-2) \sqrt[n]{|\operatorname{det}(A)|}+\lambda_{1}}
$$

from which we obtain the desired bound.

A graph $G$ is called reciprocal if for each eigenvalue $\lambda$ of $G, \frac{1}{\lambda}$ is also an eigenvalue of $G$. Therefore, for the energy of a reciprocal graph $G$, it holds:

$$
E(G)=\lambda_{1}+\left|\lambda_{2}\right|+\ldots+\left|\lambda_{n}\right|=\frac{1}{\lambda_{1}}+\frac{1}{\left|\lambda_{2}\right|}+\ldots+\frac{1}{\left|\lambda_{n}\right|} .
$$

Some energy results for the reciprocal graphs can be found in $[20,21]$. In the following result, we give a lower bound for $E(G)$ under the condition $\rho \geq 4 \mu$, where $\rho=\max \left\{\left|\lambda_{1}\right|, \ldots,\left|\lambda_{n}\right|\right\}$ and $\mu=\min \left\{\left|\lambda_{1}\right|, \ldots,\left|\lambda_{n}\right|\right\}$. 
Proposition 3. Let $G$ be a reciprocal graph on $n$ vertices, and let its eigenvalues be $\rho=\left|\lambda_{1}\right| \geq$ $\left|\lambda_{2}\right| \geq \ldots \geq\left|\lambda_{n}\right|=\mu>0$. If $\rho \geq 4 \mu$, then $E(G) \geq n+\frac{1}{2}$.

Proof. From the Cauchy-Schwarz inequality, we have:

$$
\begin{gathered}
E(G)^{2}=\left(\left|\lambda_{1}\right|+\ldots+\left|\lambda_{n}\right|\right)\left(\frac{1}{\left|\lambda_{1}\right|}+\ldots+\frac{1}{\left|\lambda_{n}\right|}\right)=\left(\left|\lambda_{n}\right|+\left|\lambda_{2}\right|+\ldots+\left|\lambda_{1}\right|\right)\left(\frac{1}{\left|\lambda_{1}\right|}+\ldots+\frac{1}{\left|\lambda_{n}\right|}\right) \geq \\
\geq\left(\sqrt{\left|\frac{\lambda_{n}}{\lambda_{1}}\right|}+\sqrt{\left|\frac{\lambda_{1}}{\lambda_{n}}\right|}+(n-2)\right)^{2}=\left(\frac{1}{\sqrt{s}}+\sqrt{s}+(n-2)\right)^{2} .
\end{gathered}
$$
$n+\frac{1}{2}$.

Since $s=\frac{\rho}{\mu} \geq 4$, we easily conclude that $\sqrt{s}+\frac{1}{\sqrt{s}} \geq \frac{5}{2}$, and consequently, $E(G) \geq$

Remark 3. We can generalize the above proposition as follows:

Let $G$ be a reciprocal graph on $n$ vertices with eigenvalues $\rho=\left|\lambda_{1}\right| \geq\left|\lambda_{2}\right| \geq \ldots \geq\left|\lambda_{n}\right|=$ $\mu>0$. If $\frac{\rho}{\mu}=s \geq 1$, then $E(G) \geq \sqrt{s}+\frac{1}{\sqrt{s}}+n-2$.

\section{Hyperenergetic and Hypoenergetic Graphs}

Let us next turn to other classes of graphs, defined via bounds on their energy. Let $G$ be a graph on $n$ vertices. The graph $G$ is called hyperenergetic if $E(G)>2 n-2$ and is called hypoenergetic if $E(G)<n$. More information about these classes of graphs can be found in [22-24]. We first show that hyperenergetic graphs trivially satisfy Conjecture 1.

Proposition 4. Let $G$ be a hyperenergetic graph with maximum degree $\Delta$ and minimum degree $\delta \geq 1$. Then:

$$
E(G) \geq \Delta+\delta .
$$

Proof. From $n-1 \geq \Delta$ and $\Delta \geq \delta$, we obtain:

$$
E(G)>2(n-1) \geq 2 \Delta \geq \Delta+\delta .
$$

Since Nikiforov proved in [25] that almost all graphs are hyperenergetic, the conjecture $E(G) \geq \Delta+\delta$ holds true for almost all graphs.

It is important to note that the nonsingularity assumption included in Conjecture 1 is essential to the potential veracity of its statement. Namely, it is not hard to find infinitely many graphs whose adjacency matrices are singular and that do not satisfy the asserted inequality. We illustrate this claim in the following example.

Example 1. Let $n \geq 4$, and let $G$ be the $n$-star graph consisting of one vertex of degree $n-1$ and $n-1$ vertices of degree 1 attached to it. Clearly, $\Delta(G)=n-1$ and $\delta(G)=1$. The spectrum of $G$ consists of the values $\{\sqrt{n-1}, 0, \ldots, 0,-\sqrt{n-1}\}$, that is the adjacency matrix of $G$ is a singular matrix [26]. None of these star graphs satisfies the inequality from Conjecture 1:

$$
\Delta(G)+\delta(G)=n-1+1=n>2 \sqrt{n-1}=E(G) .
$$

The necessity of the nonsingularity requirement is further underlined by a result of Gutman who showed in [22] that if a graph $G$ is nonsingular (i.e., no eigenvalue of $G$ is equal to zero), then $G$ is not hypoenergetic. Thus, in view of Proposition 4 , to complete the proof of Conjecture 1, one needs to focus on graphs whose adjacency matrix is nonsingular and that are neither hypoenergetic nor hyperenergetic, graphs whose energy falls in the interval:

$$
n \leq E(G) \leq 2 n-2
$$


One such class is covered in the following easy result.

Proposition 5. Let $G$ be a graph with $n$ vertices, maximum degree $\Delta$, and minimum degree $\delta=1$, and suppose that the adjacency matrix of $G$ is nonsingular. Then:

$$
E(G) \geq \Delta+\delta \text {. }
$$

Proof. Since the adjacency matrix of $G$ is assumed nonsingular, the above-mentioned result of Gutman yields $E(G) \geq n$. On the other hand, $\Delta \leq n-1$. Thus,

$$
E(G) \geq n=(n-1)+1 \geq \Delta+\delta .
$$

\section{Upper Bounds for the Energy of Graphs}

In the last section of our paper, we present an upper bound for $E(G)$ that may prove useful in disproving Conjecture 1 in its full generality. The first result of this section is an improvement on McClelland's upper bound $\sqrt{2 m n}$ [6]. Its proof relies on the same method as the proof of Theorem 2.2 in [27].

Theorem 5. Let $G$ be a graph with $n$ vertices and $m$ edges. Let $\lambda_{1} \geq \lambda_{2} \geq \ldots \geq \lambda_{n}$ be the eigenvalues of $G$, the first $p$ of which are positive, $1 \leq p<n$. Then,

$$
E(G) \leq \sqrt{2 m n-\frac{2 n}{m}\left(\lambda_{1}^{2}+\ldots+\lambda_{p}^{2}-m\right)^{2}} .
$$

Proof. From the relation in (2), $\lambda_{1}^{2}+\ldots+\lambda_{p}^{2}+\lambda_{p+1}^{2}+\ldots+\lambda_{n}^{2}=2 m$, we obtain:

$$
\begin{gathered}
\lambda_{1}^{2}+\ldots+\lambda_{p}^{2}-m=\frac{1}{2}\left(\lambda_{1}^{2}+\ldots+\lambda_{p}^{2}-\left(\lambda_{p+1}^{2}+\ldots+\lambda_{n}^{2}\right)\right)= \\
=\frac{1}{2}\left(\lambda_{1}\left|\lambda_{1}\right|+\lambda_{2}\left|\lambda_{2}\right|+\ldots+\lambda_{n}\left|\lambda_{n}\right|\right) .
\end{gathered}
$$

The required inequality in (20) is equivalent to:

$$
\frac{n}{E(G)^{2}} \geq \frac{2 m}{4 m^{2}-\left(\lambda_{1}\left|\lambda_{1}\right|+\ldots+\lambda_{n}\left|\lambda_{n}\right|\right)^{2}} .
$$

By applying $\lambda_{1}+\ldots+\lambda_{n}=0$ and $\lambda_{1}^{2}+\ldots+\lambda_{n}^{2}=2 m$, we obtain the following identity:

$$
\begin{gathered}
\frac{2 m}{4 m^{2}-\left(\lambda_{1}\left|\lambda_{1}\right|+\ldots+\lambda_{n}\left|\lambda_{n}\right|\right)^{2}}=\sum_{i=1}^{n}\left(\frac{2 m\left|\lambda_{i}\right|-\left(\lambda_{1}\left|\lambda_{1}\right|+\ldots+\lambda_{n}\left|\lambda_{n}\right|\right) \lambda_{i}}{4 m^{2}-\left(\lambda_{1}\left|\lambda_{1}\right|+\ldots+\lambda_{n}\left|\lambda_{n}\right|\right)^{2}}\right)^{2}= \\
=\sum_{i=1}^{n} \frac{1}{E(G)} \cdot \frac{2 m\left|\lambda_{i}\right|-\left(\lambda_{1}\left|\lambda_{1}\right|+\ldots+\lambda_{n}\left|\lambda_{n}\right|\right) \lambda_{i}}{4 m^{2}-\left(\lambda_{1}\left|\lambda_{1}\right|+\ldots+\lambda_{n}\left|\lambda_{n}\right|\right)^{2}}
\end{gathered}
$$

In the end, we obtain:

$$
\begin{gathered}
\frac{n}{E(G)^{2}}-\frac{2 m}{4 m^{2}-\left(\lambda_{1}\left|\lambda_{1}\right|+\ldots+\lambda_{n}\left|\lambda_{n}\right|\right)^{2}}=\sum_{i=1}^{n} \frac{1}{E(G)^{2}}-\sum_{i=1}^{n}\left(\frac{2 m\left|\lambda_{i}\right|-\left(\lambda_{1}\left|\lambda_{1}\right|+\ldots+\lambda_{n}\left|\lambda_{i}\right|\right) \lambda_{i}}{4 m^{2}-\left(\lambda_{1}\left|\lambda_{1}\right|+\ldots+\lambda_{n}\left|\lambda_{n}\right|\right)^{2}}\right)^{2}= \\
=\sum_{i=1}^{n}\left(\frac{1}{E(G)}-\frac{2 m\left|\lambda_{i}\right|-\left(\lambda_{1}\left|\lambda_{1}\right|+\ldots+\lambda_{n}\left|\lambda_{n}\right|\right) \lambda_{i}}{4 m^{2}-\left(\lambda_{1}\left|\lambda_{1}\right|+\ldots+\lambda_{n}\left|\lambda_{n}\right|\right)^{2}}\right)^{2} \geq 0 .
\end{gathered}
$$


Corollary 1. Let $G$ be a graph with $n$ vertices, $m$ edges, and exactly one positive eigenvalue. Then:

$$
E(G) \leq 2 \sqrt{m+m \sqrt{\frac{n-2}{n}}} .
$$

Proof. Let $n$ be the order of $G$ and $\rho$ be the largest (positive) eigenvalue of $G$. Applying Theorem 5, we obtain:

$$
E(G) \leq \sqrt{2 m n-\frac{2 n}{m}\left(\rho^{2}-m\right)^{2}}
$$

Using (22) and $E(G) \geq 2 \sqrt{m}$, we obtain the inequality $4 m \leq 2 m n-\frac{2 n}{m}\left(\rho^{2}-m\right)^{2}$, which is equivalent to:

$$
\rho \leq \sqrt{m+m \sqrt{\frac{n-2}{n}}} .
$$

Since $E(G)=2\left(\lambda_{1}+\ldots+\lambda_{p}\right)$, where $\lambda_{1}, \ldots, \lambda_{p}$ are the positive eigenvalues of $G$, we obtain:

$$
E(G)=2 \lambda_{1}=2 \rho \leq 2 \sqrt{m+m \sqrt{\frac{n-2}{n}}} .
$$

Remark 4. One can check that starting from $n>4$, the relation in Corollary 1 is tighter than McClelland's $E(G) \leq \sqrt{2 m n}$. For these cases,

$$
E(G)^{2} \leq 4 m\left(1+\sqrt{\frac{n-2}{n}}\right)<8 m<2 m n .
$$

In the last result, we compare our bound (21) with the Koolen and Moulton bound (5).

Proposition 6. Let $G$ be a graph with $n$ vertices, $m(>8)$ edges, and exactly one positive eigenvalue. If $n \in\left(\frac{5+\sqrt{25+8 m-16 \sqrt{2 m}}}{2}, 2 m\right]$, then:

$$
2 \sqrt{m+m \sqrt{\frac{n-2}{n}}}<\frac{2 m}{n}+\sqrt{(n-1)\left(2 m-\frac{4 m^{2}}{n^{2}}\right)} .
$$

Proof. Since $m \sqrt{\frac{n-2}{n}}<m$, it suffices to prove that:

$$
2 \sqrt{2 m}<\frac{2 m}{n}+\sqrt{(n-1)\left(2 m-\frac{4 m^{2}}{n^{2}}\right)} .
$$

From $2 m \leq n \Delta<n^{2}$, we have $n>\sqrt{2 m}$. Thus, $2 n \sqrt{2 m}>2 \sqrt{2 m} \sqrt{2 m}=4 m>2 m$, which leads to $2 \sqrt{2 m}-\frac{2 m}{n}>0$. Now, we have:

$$
\begin{gathered}
2 \sqrt{2 m}<\frac{2 m}{n}+\sqrt{(n-1)\left(2 m-\frac{4 m^{2}}{n^{2}}\right)} \Leftrightarrow\left(2 \sqrt{2 m}-\frac{2 m}{n}\right)^{2}<(n-1)\left(2 m-\frac{4 m^{2}}{n^{2}}\right) \Leftrightarrow \\
f(n)=n^{2}-5 n+4 \sqrt{2 m}-2 m>0 . \\
\text { From } D(f)=25+8 m-16 \sqrt{2 m} \geq 2 \sqrt{25 \cdot 8 m}-16 \sqrt{2 m}=4 \sqrt{2 m}>0, \text { we obtain: }
\end{gathered}
$$

$$
f(n)>0 \Leftrightarrow n \in\left(-\infty, \frac{5-\sqrt{25+8 m-16 \sqrt{2 m}}}{2}\right) \cup\left(\frac{5+\sqrt{25+8 m-16 \sqrt{2 m}}}{2}, \infty\right) .
$$


For $m>8$, the left interval in (23) contains only negative numbers. Since for a connected graph with $n$ vertices and $m$ edges, it holds $n \in(\sqrt{2 m}, 2 m]$, we conclude that:

$$
2 \sqrt{2 m}<\frac{2 m}{n}+\sqrt{(n-1)\left(2 m-\frac{4 m^{2}}{n^{2}}\right)} \Leftrightarrow n \in\left(\frac{5+\sqrt{25+8 m-16 \sqrt{2 m}}}{2}, 2 m\right] .
$$

Author Contributions: All the authors contributed equally, and they read and approved the final manuscript for publication. All authors read and agreed to the published version of the manuscript.

Funding: The first author is supported by the Slovenian Research Agency (Research Project P1-0285). The second author is supported by VEGA 1/0596/17, VEGA 1/0719/18, VEGA 1/0423/20, and APVV-15-0220 and by the Slovenian Research Agency (Research Projects N1-0038, N1-0062, J1-9108).

Data Availability Statement: Not applicable.

Conflicts of Interest: The authors declare that they have no competing interests.

\section{References}

1. Gutman, I. The energy of a graph, 10. Steiermärkisches Mathematisches Symposium (Stift Rein, Graz, 1978). Ber. Math. Stat. Sekt. Forschungszent. Graz 1978, 103, 1-22.

2. Gutman, I. The energy of a graph: Old and new results. In Algebraic Combinatorics and Applications; Betten, A., Kohnert, A., Laue, R., Wassermann, A., Eds.; Springer: Berlin/Heidelberg, Germany, 2001; pp. 196-211.

3. Gutman, I.; Polansky, O.E. Mathematical Concepts in Organic Chemistry; Springer: Berlin/Heidelberg, Germany, 1986.

4. Gutman, I. On graphs whose energy exceeds the number of vertices. Linear Algebra Appl. 2008, 429, 2670-2677. [CrossRef]

5. Gutman, I.; Li, X.; Zhang, J. Graph energy. In Analysis of Complex Networks; Dehmer, M., Emmert-Streib, F., Eds.; From Biology to Linguistics; Wiley-VCH: Weinheim, Germany, 2009; pp. 145-174.

6. McClelland, B.J. Properties of the latent roots of a matrix: The estimation of $\pi$-electron energies. J. Chem. Phys. 1971, 54, 640-643 [CrossRef]

7. Babic, D.; Gutman, I. More lower bounds for the total $\pi$-electron energy of alternant hydrocarbons. MATCH Commun. Math. Comput. Chem.1995, 32, 7-17.

8. Gutman, I.; Teodorovic, A.V.; Nedeljkovic, L. Topological properties of benzenoid systems. Bounds and approximate formula for total $\pi$-electron energy. Theor. Chim. Acta 1984, 65, 23-31. [CrossRef]

9. Jahanbani, A. Some new lower bounds for energy of graphs. Appl. Math. Comput. 2017, 296, 233-238. [CrossRef]

10. Koolen, J.H.; Moulton, V. Maximal energy graphs. Adv. Appl. Math. 2001, 26, 47-52. [CrossRef]

11. Li, X.; Shi, Y.; Gutman, I. Graph Energy; Springer: New York, NY, USA, 2010.

12. Caporossi, G.; Cvetkovic, D.; Gutman, I.; Hansen, P. Variable Neighborhood Search for Extremal Graphs. 2. Finding Graphs with Extremal Energy. J. Chem. Inf. Comput. Sci. 1999, 39, 984-996. [CrossRef]

13. Ma, X. A low bound on graph energy in terms of minimum degree. MATCH Commun. Math. Comput. Chem. 2019, 81, 393-404.

14. Akbari, S.; Hosseinzadeh, M.A. A short proof for graph energy is at least twice of minimum degree. MATCH Commun. Math. Comput. Chem. 2020, 83, 631-633.

15. Oboudi, M.R. A very short proof for a lower bound for energy of graphs. MATCH Commun. Math. Comput. Chem. 2020, 84, 345-347.

16. Akbari, S.; Ghahremani, M.; Hosseinzadeh, M.A.; Ghezelahmad, S.K.; Rasouli, H.; Tehranian, A. A Lower Bound for Graph Energy in Terms of Minimum and Maximum Degrees. MATCH Commun. Math. Comput. Chem. 2019, 81, 393-404.

17. Edwards, C.S. The largest vertex degree sum for a triangle in a graph. Bull. London Math. Soc. 1977, 9, 203-208. [CrossRef]

18. Ilic, A.; Liu, B. On the upper bounds for the first Zagreb index. Kragujev. J. Math. 2011, 35, 173-182.

19. Mildorf, T.J. Olympiad Inequalities. 2005. Available online: https://artofproblemsolving.com/articles/files/MildorfInequalities. pdf (accessed on 16 May 2021).

20. Indulal, G.; Vijayakumar, A. Reciprocal graphs. Malaya J. Mat. 2016, 4, 380-387.

21. Filipovski, S.; Jajcay, R. New upper bounds for the energy and spectral radius of graphs. MATCH Commun. Math. Comput. Chem. 2020, 84, 335-343.

22. Gutman, I. Hyperenergetic and Hypoenergetic Graphs, Zbornik Radova. In Selected Topics on Applications of Graph Spectra; Matematicki Institut SANU: Beograd, Serbia, 2011; pp. 113-135.

23. Gutman, I. Hyperenergetic molecular graphs. J. Serbian Chem. Soc. 1999, 64, 199-205.

24. Walikar, H.B.; Ramane, H.S.; Hampiholi, P. On the energy of a graph. In Graph Connections; Balakrishnan, R., Mulder, H.M., Vijayakumar, A., Eds.; Allied Publishers: New Delhi, India, 1999; pp. 120-123.

25. Nikiforov, V. The energy of graphs and matrices. J. Math. Anal. Appl. 2007, 326, 1472-1475. [CrossRef] 
26. Jones, O. Spectra of Simple Graphs. 2013. Available online: https://www.whitman.edu/Documents/Academics/Mathematics/ Jones.pdf (accessed on 16 May 2021).

27. Filipovski, S. New upper bounds for the first Zagreb index. MATCH Commun. Math. Comput. Chem. 2015, 74, 97-101. 